\title{
A Simplified Analytical Model Toward Big Data Analysis using Ridge Regression Method
}

\author{
Afreen Ali \\ Computer Science \& Engineering, Department \\ All Saint's College of Technology \\ Bhopal, India
}

\author{
Sarwesh Site \\ Computer Science \& Engineering, Department \\ All Saint's College of Technology \\ Bhopal, India
}

\begin{abstract}
Ridge Regression is a essential method in linear regression used to analyze multiple regression data which have multicollinearity. For solving highly related multicollinearity problems, Ridge Regression is a better modeling technique than ordinary least square method. The analytical data in modern technology is becoming extremely large in size and the term which describes this large volume of data is "Big Data", and ordinary tools are insufficient to analyzes big data. In this paper, we are presenting an approach toward big data analysis through ridge regression method. Our simulation result represents a mapping model of Gaussian data from big data in sufficient scale. This model presents the new gateway for big data for statistical and mathematical analysis.
\end{abstract}

\section{General Terms}

Ridge Regression

\section{Keywords}

Big Data, MapReduce, Statistics, Regression Model, Gaussian Data

\section{INTRODUCTION}

Ridge regression is used as a part of highly correlated multiindependent factor related dependent variables. It is worked to reduce the effect of all factor on the any other. It is a process for examining multiple regression data that suffer from multicollinearity [1]. When multicollinearity happens, least squares estimates are unbiased, but their differences are large so their may be far to the true value. From computing a degree of bias to the regression approximations, ridge regression undermine the standard errors [2]. The world has come into the era of big data in any case, more and more big data issues are deriving in fields, such as scientific research, international economics, public administration and so on. Discovering techniques to big data became a current eventuality and challenge. In big data applications, it is usual for the attribute to work in the same manner with response or explanatory variable [3]. Various techniques of analysis was developed in view of such problems, like logistic regression, and $k$-nearest neighbor method so on. In any way, some research express an important type of big data issue in which the response variable works as the real numeric type and the explanatory variable is the attribute type [4].
In ordinary least squares, the regression coefficients are estimated represented in formula

$$
\underline{\hat{\mathbf{B}}}=\left(\mathbf{X}^{\prime} \mathbf{X}\right)^{-1} \mathbf{X}^{\prime} \underline{\mathbf{Y}}
$$

Note that fact since then the variables are standardized, $\mathbf{X}^{\prime} \mathbf{X}=\mathbf{R}$, where $\mathbf{R}$ is the correlation matrix of independent variables. These approximations are unbiased so that the expected value of the estimates are the population values. That is,

$$
E(\underline{\hat{\mathbf{B}}})=\underline{\mathbf{B}}
$$

The variance-covariance matrix of the estimations is

$$
V(\underline{\hat{\mathbf{B}}})=\sigma^{2} \mathbf{R}^{-1}
$$

and since then we are supposing that the $y^{\prime}$ s are standardized, $\sigma^{2}=1$. From the above, we find that

$$
V\left(\hat{b}_{j}\right)=r^{j j}=\frac{1}{1-R_{j}^{2}}
$$

where $R_{j}^{2}$ is the $R$-squared value obtained from regression $X_{j}$ on the other independent variables. Ridge regression advances by including a small value, $k$, to the diagonal elements for the correlation matrix. This is the place ridge regression gets its name since the diagonal of ones in the correlation matrix might be consideration of as a ridge.

That is shown as,

$$
\underline{\tilde{\mathbf{B}}}=(\mathbf{R}+k \mathbf{I})^{-1} \mathbf{X}^{\prime} \underline{\mathbf{Y}}
$$

where, $k$ is a positive amount less than one.

The measure of bias in this estimator is predicted by

$$
E(\underline{\tilde{\mathbf{B}}}-\underline{\mathbf{B}})=\left[\left(\mathbf{X}^{\prime} \mathbf{X}+k \mathbf{I}\right)^{-1} \mathbf{X}^{\prime} \mathbf{X}-\mathbf{I}\right] \underline{\mathbf{B}}
$$

and the covariance matrix is given by

$$
V(\underline{\tilde{\mathbf{B}}})=\left(\mathbf{X}^{\prime} \mathbf{X}+k^{\mathbf{I}}\right)^{-1} \mathbf{X}^{\prime} \mathbf{X}\left(\mathbf{X}^{\prime} \mathbf{X}+k \mathbf{I}\right)^{-1}
$$

It can be shown that there consists a value of $k$ for which the mean squared error (the variance plus the bias squared) of the ridge estimator is less than that of the least squares estimator [5]. Unfortunately, the appropriate value of $k$ depends on knowing the true regression coefficients which are estimated and an analytic solution has not been discovered which ensures the optimality of the ridge solution [6]. 


\subsection{MapReduce}

MapReduce is a system using that the applications can be composed to process enormous magnitude of data, in similar, on major clusters of objective hardware in a determined manner [7][8]. M. R. Thakare et al. [9] presented the master node having the input that classify data into minor sub parts and partition in worker nodes. It is a software development model which is inspired by functional programming and permits expressing distributed calculations on extensive amounts of data [10].

MapReduce is a method to process massive amounts of data, in parallel, on large sets of commodity hardware in a dependable manner [11]. It is a software development model and a related implementation for generating and processing large data sets. The distributed data processing feature by Apache has been used for mining really massive datasets [12].

MapReduce as a model of programming can be understandable as a method which is executed to process big data, by the usage of a distributed and parallel running calculation in a groups [13]. It is a handling technique and a program show for distributed computing depended on java. The MapReduce algorithm consists of two essential task, that is Map and Reduce [14]. Map takes a collection of data and converts it into another collection of data, where individual elements are separated into tuples (key/value pairs) [2][15]. Also, reduce task, which takes the output from a map as an input and joins those data tuples into a smaller collection of tuples. As the arrangement of the name MapReduce suggests, the reduce task is constantly performed after the map job [16].

\subsection{Decision Tree}

A decision tree is a tool of a tree-like graph of decisions and their conceivable outcomes, including chance event results, and utility. B. Gupta et al. [17] suggested a way to view an algorithm that exclusive contains conditional control statements. It influences uses of recursive tree to structure and is a consecutive classifier. There are three kinds of nodes in the decision tree. The node from which the tree is coordinated and has no approaching edge is known as the root node. A node with dynamic edge is called internal or test node however the different nodes are called leaves (also known as terminal or decision node). The data set in decision tree is examined by developing a branch like structure with proper decision tree algorithm. Each internal node of tree parts into branches in view of the splitting criteria. Each test node signifies a class. Each terminal node represents the decision. They can work for categorical and continuous properties.

A decision tree for the concept check 'Humidity', indicating whether a checking humidity is shown in Figure 1 Each internal node represents a test on an attribute. Each external node represents a class (either indicate the humidity is high $=$ "no" or normal = "yes").

\subsection{Types of Decision Trees}

Decision trees applied in data mining are of two types:

- Classification Tree in which analysis is when the predicted outcome is the class to which the data belongs. For example outcome of loan application as safe or risky.

- Regression Tree in which analysis is when the predicted outcome can be considered a real number. For example population of a state.

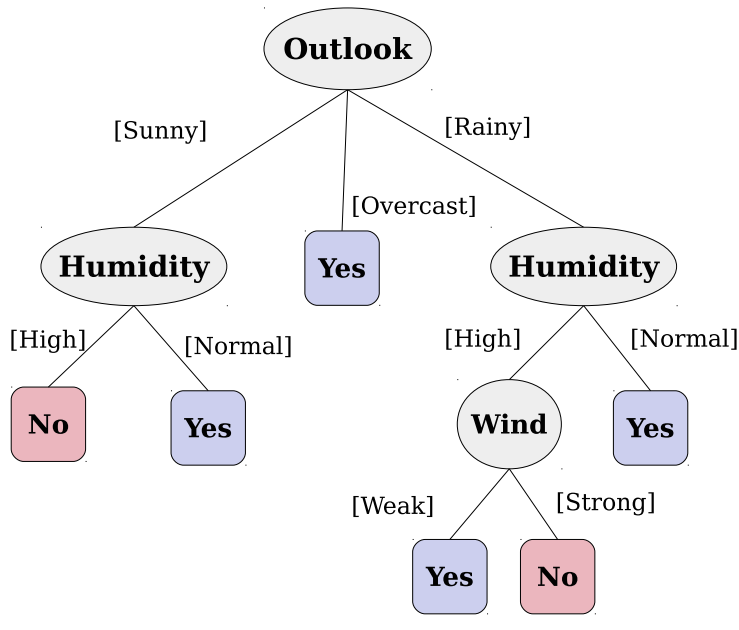

Fig. 1. A Decision Tree for The Concept of Check The Weather

\subsection{Big Data Research}

In the current digitally connected world, each and every thing can be examined of creating data. The terminology Big Data is not just about the importance of data that comes in the scope of MapReduce is considered a simplification approach for parallel computation of large data [18]. The MapReduce implementation depends on specific or customized cluster management that is responsible for distributing and running peta bytes or zeta bytes something it is more about the capability to consider large amounts of data. This data that is getting added to the current ocean of Big Data formed from uncountable sources like web logs, PDAs, smart phones, social network sites, satellite pictures, biological records, client exchanges and astronomical, poses immense opportunities as well as challenges for researchers to handle and provide useful result. S. Jun et al. [19] proposed an approach to overcome the computing burden in big data analysis because most statistical methods were determined on small sample data. Also in big data analysis, it analyze entire data which are considered as population in statistics, and this data set is so huge. This research divided the big data closed to population into sub data set like sample for solving the computing cost in big data analysis. In addition, they applied this approach to regression problem in statistics. They applied the divided method of big data to multiple regression analysis, and used simple random sampling for big data dividing. Big data have also these four fields. Big data analysis is one of big data science, and statistics support key performance to the big data analysis. Figure 2 presents a relation of statistics to big data.

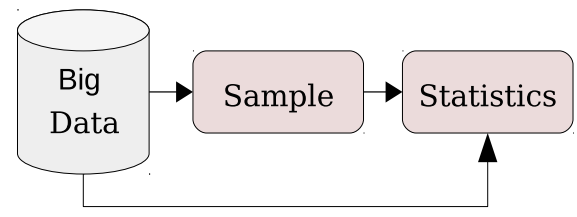

Fig. 2. From Big Data to Statistics

First, sample are extracted from the big data, and then the sample are analyzed using statistical methods. This is the traditional approach of statistical analysis. In this process, big data is considered 
as a population. In statistics, a population is defined as a set of total elements in the subject of study, and the population cannot be analyzed because of its analyzing cost or changeable. But in big data, a data set can be analyzed closed to the population. It is caused by the development of computing large data and decreasing the price of data storage. But, the computing burden of big data analysis remains because the traditional analyses such as statistical methods have a limitation for analyzing big data.

L. R. Nair et al. [20] presented the research of in Big Data progresses in different dimensions including effective capture of data, discovering novel storage solutions and recovery techniques. Figure 3 gives an outline of Big Data analytics flow. Also, much consideration has been given to Big Data analytics in cloud environment. Social network source big data analytics is also a dynamic area of research to discover cutting edges. Bo-Wei Chen et al. [21] presented Big Data analytic framework for scalable machine learning, data mining algorithms, exact learning, accurate predictions, developing new representation techniques, receiving tight security, privacy preservation strategies and benchmarking.

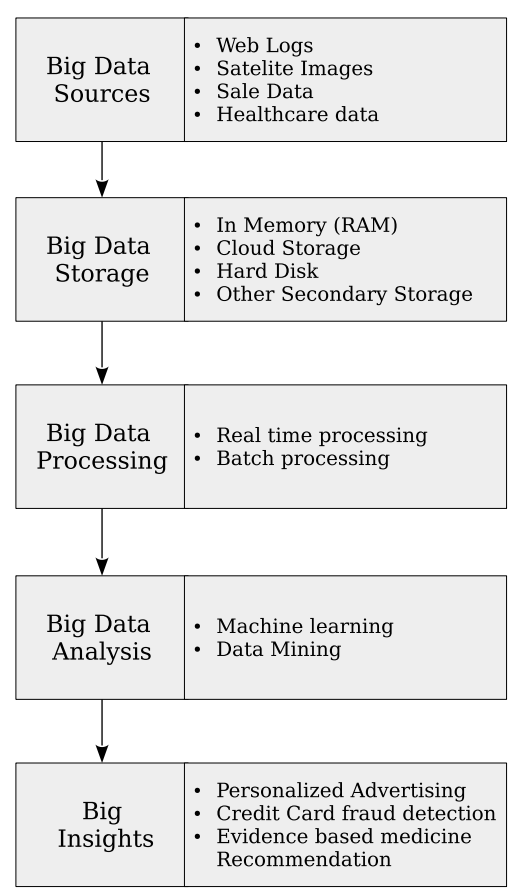

Fig. 3. Big Data Analytic Flow

\section{BIG DATA TECHNOLOGIES}

Big data technologies are important in giving more precise analysis, which may lead to more concrete decision-making resulting in greater operational efficiencies, cost reductions and reduced risks for the business. There are different technologies in the market from various vendors including Amazon, IBM, Microsoft to deal with big data which look into the technologies that handle big data. Goldar et al. [22] presented realistic tools, techniques and methods for parallelization of big data such as Hadoop, MongoDB and Spark, the description of these tools are as follows:

\subsection{Hadoop}

Hadoop [23][24] is an Apache open source system composed in java that permits distributed processing of large datasets across clusters of computers operating basic programming models. A Hadoop frame-worked application works in a space that gives distributed storage and computation across clusters of computers. Hadoop is intended to scale up from single server to an expansive number of machines, each offering nearby computation and storage capacity. The working flow of big data parallelization under Hadoop is represented in Figure 4

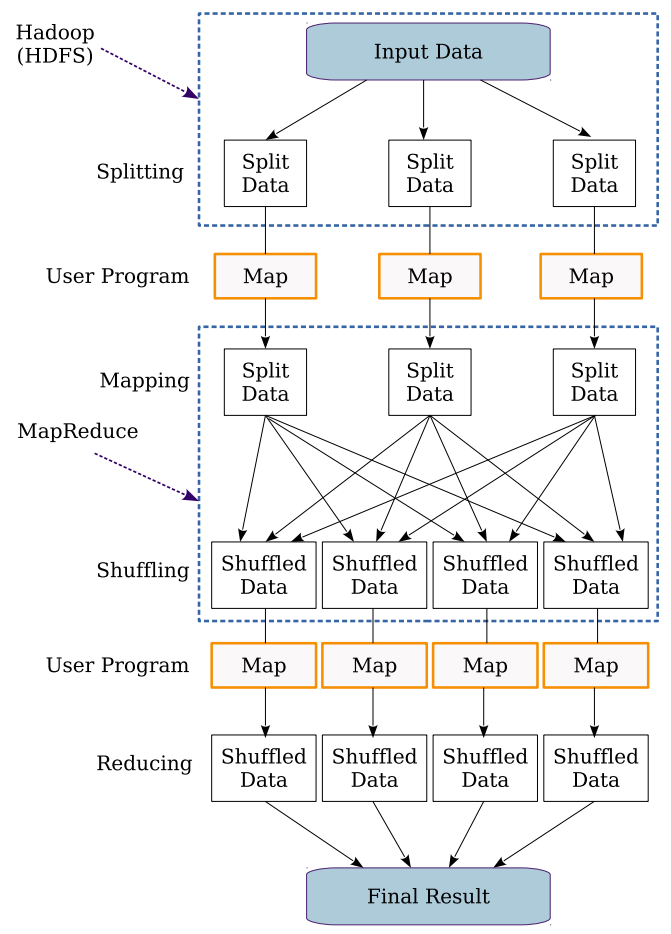

Fig. 4. Hadoop

2.1.1 Hadoop MapReduce. Hadoop MapReduce is a software framework for easily composing applications which process big amounts of data in-parallel on large clusters (a huge number of nodes) of commodity hardware in a dependable, fault-tolerant manner [25]. The term MapReduce really refers to the following two other tasks that Hadoop programs perform:

- The Map Task This is the primary task, which takes input information and converts it into a collection of data, where individual elements are separated into tuples.

- The Reduce Task This task takes the output from a map task as input and joins those data tuples into a smaller collection of tuples. The reduce task is always performed after the map task.

2.1.2 Hadoop Distributed File System. The software for dataintensive parallel and distributed applications, based on MapReduce programming framework and the distributed file system is called Hadoop Distributed Filesystem (HDFS) [26]. HDFS is now a sub-project of Apache Hadoop project. It is designed to supply the fault-tolerant file system to run on initial hardware. The principal intention of HDFS is to collect data reliably, which works even 
in the occurrence of system failures. HDFS uses the master/slave framework where one master device controls one or more other slave devices.

The Hadoop Distributed File System (HDFS) depends on the Google File System (GFS) and gives a distributed file system that is intended to running on large clusters (thousands of computers) of small computer machines in a reliable, fault-tolerant manner. A document in an HDFS namespace is part into few blocks and those blocks are stored in a set of DataNodes. The NameNode decides the mapping of blocks to the DataNodes. The DataNodes deal with read and write operation with the file system. They additionally deal with block creation, deletion and replication in view of direction given by NameNode.

HDFS gives a shell like some other file system and a list of commands are accessible to cooperate with the file system.

\subsection{MongoDB}

MongoDB [27][28] is a cross-platform, document oriented database that gives, high performance, and easy scalability. MongoDB works on concept of collection and document. The structure of MongoDB is presented in Figure 5

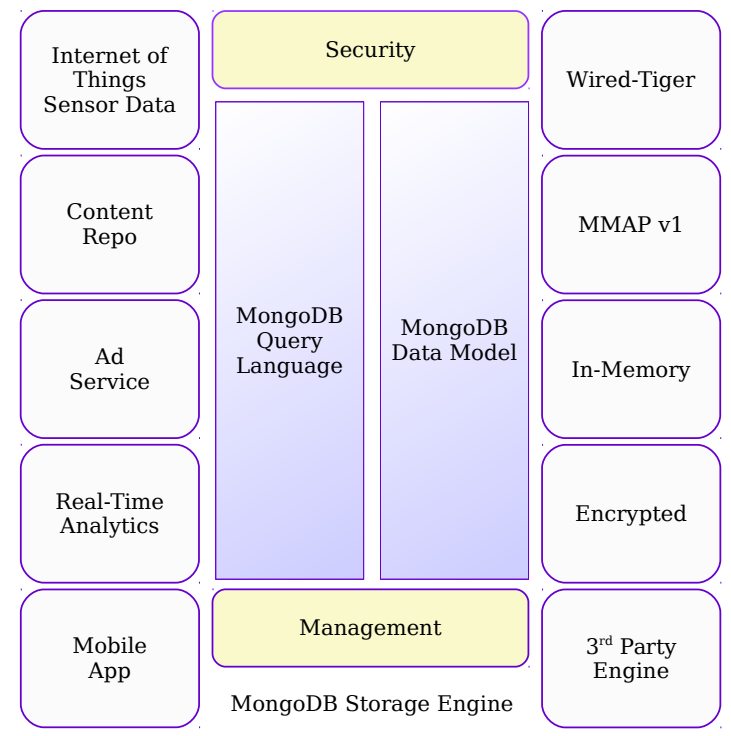

Fig. 5. MongoDB

\footnotetext{
-Database Database is a physical container for collections. Every database gets its own particular arrangement files on the file system. A single MongoDB server regularly has multiple databases.

- Collection Collection is a group of MongoDB records. It is the equivalent of an RDBMS table. A collection exists inside a single database. Collections don't authorize a pattern. Documents within a collection can have different fields. Normally, all documents in a collection are of similar or related purpose.

-Document A document is an arrangement of key-value pairs. Documents have dynamic pattern. Dynamic schema implies that documents in a similar collection don't need to have the same set of fields or structure, and common fields in a collection's documents may hold different types of data.
}

\subsection{Spark}

Industries are using Hadoop broadly to examine their data sets. The reason is that Hadoop structure depends on a basic programming model (MapReduce) and it enables a computing solution that is scalable, fault-tolerant and cost effective. Here, the fundamental concern is to keep up speed in processing extensive datasets in terms of waiting time between queries and holding up time to run the program.

Spark was presented by Apache Software Foundation for speeding up the Hadoop computational computing software process. Spark uses Hadoop in two ways one is storage and second is processing. Since Spark has its own cluster management computation, it works Hadoop for storage.

Apache Spark. Apache Spark [29] is a lightning-fast cluster computing technology, intended for fast computation. It depends on Hadoop MapReduce and it extends the MapReduce model to effectively use it for more types of computations, which includes interactive queries and stream processing. The principle of Spark is its in-memory cluster computing that increases the processing speed of an application. Spark is intended to cover an extensive range of workloads for example batch applications, iterative algorithms, interactive queries and streaming. Aside from supporting all these workload in a particular system, it decreases the administration burden of maintaining separate tools. The structure of Spark is presented in Figure 6.

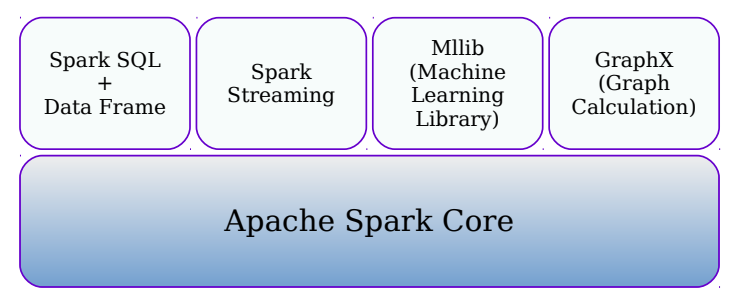

Fig. 6. Spark

\section{RELATED WORK}

\subsection{An exact approach to ridge regression for big data}

Zhang et al. [30] proposed a ridge regression approach to big data. Ridge regression is important and has been considerably used in applications. The classical ridge regression approach centers around on small or moderate data. It accept that the whole data set can be loaded to the memory of a personal computer. However, if the data collection is large, at that it cannot be loaded to memory, which implies that the traditional approach cannot be used. To solve the issue, they proposed new techniques and algorithms, where the whole data set is only scanned once. The objective of scanning data is to register a matrix of sufficient statistics [31], which is not expansive. Once the matrix of sufficient statistics is derived, the greater part of the rest computations can be totally done without the need of the first data set. Therefore, our numerical algorithms are efficient. Uses of ridge regression are still outstanding in many research areas. Cases include pattern and face recognition described by Xue $e t$ al. [32] in 2009, genetics, and machine learning. Previous methods and algorithms for ridge regression are for the most part produced for small or, on the other hand again moderate data. They cannot be used to examine big data as a result of the presence of memory and computational efficiency barriers. 
Ridge regression has turned to be popular and all around acknowledged since it was first proposed by Hoerl and Kennard et. al [33] in 1970. At the point when multicollinearity happens, despite the fact that the standard thing least squares estimators are as yet unbiased, their variances are absolutely inflated. By including a small degree of bias to the least squares estimators, ridge regression can significantly reduce their standard errors and in this manner increment the levels of significance. The aim of the present article is to propose new methods and algorithms for ridge regression which can overcome these challenges. Ridge regression is one of the extremely common techniques to increase the power [34].

\subsection{Classical Ridge Regression}

R. L. Obenchain et al. [35] proposed a method For testing general linear speculations in multiple regression models. it is demonstrated that non-stochastically shrunken ridge estimators yield a similar central F-ratios and t-statistics as does the least squares estimator. Thus although ridge regression does produce biased point estimates which deviate from the least squares solution, ridge techniques do not generally yield "new" normal theory statistical inferences: in particular, ridging does not necessarily produce shifted confidence regions. A concept, the "ASSOCIATED PROBABILITY" of a ridge estimate, is defined using the standard, hyperellipsoidal confidence region centered at the least squares estimator, and it is contended that ridge estimates are of moderately little interest when they are so extreme to the point that they lie outside of the least squares region of say 90 percent confidence.

Recently, the term big data has advance quickly in both statistics and computer science. When handling with statistical methods for big data, computing the approximation of model parameters via traditional methods and algorithms is impossible because they attempt to load the total data set to the memory, indicating that neither the memory barrier nor the computational efficiency barrier is considered. To conquer these difficulties, new reasoning in statistics and computer science is required Fan et al. [36] 2014. Traditional algorithms perform well only in moderate data. If the entire data set is stacked to memory of a computer, at that point standard algorithms can be connected. Examples incorporate the computation of the rank statistics, the order statistics, and the standardization 37]. $\mathrm{Be}$ that as it may, these algorithms are infeasible in big data.

\subsection{Regression Model for Big Data with Attributive Explanatory Variables}

Qing-Ting et al. [38] proposed using the SLR method to address big data problems of linear relation. Compared with the direct estimation method, which requires extensive historical sample data, our SLR method uses limited storage space, even as the data size grows. In addition, the sequential updating process is extremely efficient. As the applications for modeling of big data and analysis advance in scope, computational efficiency faces greater challenges in terms of storage and speed. In many practical problems, a great amount of historical data is sequentially collected and used for online statistical modeling. For modeling sequential data, It is a sequential linear regression method that extracts essential information from historical data. This carefully selected information is then utilized to update a model according to a sequential estimation scheme. With this technique, the earlier data no longer needs to be stored, and the sequential updating is computationally efficient in speed and storage. A weighted strategy is introduced on the current model to determine the impact of data from different periods. When compared with estimation methods that use historical data, our numerical experiments demonstrate that our solution increased the speed while decreasing the storage load.

\subsection{Statistics in Data Mining}

A research by J. H. Friedman et al. [39], explains the connection between Statistics and Data Mining. Statistics is the study of collection, analysis, interpretation, presentation, and organization of data. Statistics is a component of data mining that provides the tools and analytics techniques for dealing with large amounts of data. It is the science of learning from data and includes everything from collecting and organizing to analyzing and presenting data. It is concerned with probabilistic models, specifically inference, using data [40]. Figure 7] represents relation of data mining to other disciplines of computer science and mathematics. While the aims

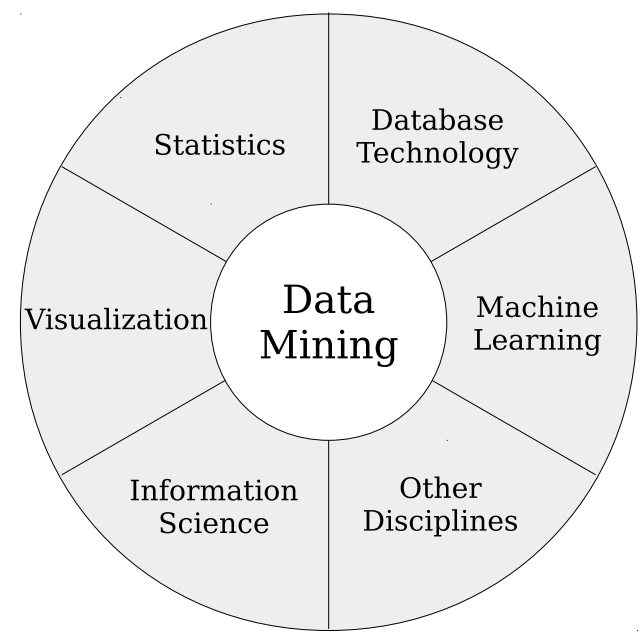

Fig. 7. Statistics in Data Mining

of statistics and data mining are similar, it is estimated that there are very few statisticians to deal with the demands of data analysts [41]. Data mining and statistics are related to learning from data. They are all about discovering and identifying structures in them, thus aimed at turning data to information. And although the aims of both these techniques overlap, they have different approaches. Statistics is only about quantifying data. While it uses tools to find relevant properties of data, it is a lot like math. It provides the tools necessary for data mining [42].

The two main statistical methodologies are descriptive statistics and inferential statistics. Descriptive statistics organize and summarize the data for the sample. The methodology of using these summaries to draw conclusions from entire data sets, is called inferential statistics.

\section{PROPOSED APPROACH}

The sufficient size should be estimated of data set according to its available computing resources. As described by Emerson et al. [43], a data set is considered as 'large' if it consumes $20 \%$ of random access memory (RAM) on a single processor computer and 'massive' if it reach out $50 \%$. Generally, the volume of big data is much larger than the storage volume of a single computer disk, the input data is stored in multiple disks. The computational task is distributed among many parallel computers so the job is finished 
in a sensible amount of time. A method and algorithm based on a single processor system is presented for big data analysis.

The proposed method is an approach for the computation of the exact ridge regression parameters for big data. The approach contains statistical methods and numerical algorithms for $\hat{\beta}_{s, \lambda}$ and $\hat{V}\left(\hat{\beta}_{s, \lambda}\right)$ with respect a single processor system. The entire data set cannot be loaded to the memory but can be saved to the hard disk of a computer, so it is assumed that any standard treatment procedure of the raw data cannot be applied because the results from these procedures must be saved either to the memory or the hard disk, which is infeasible.

In order to propose an efficient method for ridge regression to big data, the properties of the likelihood function is considered. Based on the distribution of $\epsilon_{i}$, the $\log$ likelihood function is

$$
\ell\left(\beta, \sigma^{2}\right)=-\frac{n}{2} \log (2 \pi)-\frac{n}{2} \log \sigma^{2}-\frac{1}{2 \sigma^{2}} \sum_{i=1}^{n}\left(y_{i}-\mathbf{x}_{i}^{\prime} \beta\right)^{2}
$$

The maximum likelihood estimation (MLE) of $\beta$ is

$$
\hat{\beta}=\left(\sum_{i=1}^{n} \mathbf{x}_{i} \mathbf{x}_{i}^{\prime}\right)^{-1}
$$

and the maximum likelihood estimation (MLE) of $\sigma^{2}$ is

$$
\hat{\sigma^{2}}=\frac{1}{n}\left[\sum_{i=1}^{n} y_{i}^{2}-\left(\sum_{i=1}^{n} \mathbf{x}_{i} y_{i}\right)^{\prime}\left(\sum_{i=1}^{n} \mathbf{x}_{i} \mathbf{x}_{i}^{\prime}\right)\left(\sum_{i=1}^{n} \mathbf{x}_{i} y_{i}\right)\right]
$$

Observing the right sides of Equation 9 and 10 we find that $\hat{\beta}$ and $\hat{\sigma^{2}}$ can be expressed as functions of $s_{y y}=\sum_{i=1}^{n} y_{i}{ }^{2}, S_{x y}=\sum_{i=1}^{n} \mathbf{x}_{i} y_{i}$ and $\mathbf{S}_{x x}=\sum_{i=1}^{n} \mathbf{x}_{i} \mathbf{x}_{i}^{\prime}$ where $s_{y y}$ is a univariate quantity, $s_{x y}$ is a $p-$ dimensional vector, and $S_{x x}$ is a $p \times p-$ dimensional matrix. Then,

$$
\hat{\beta}=\mathbf{S}_{x x}^{-1} \mathbf{s}_{x y}
$$

and

$$
\hat{\sigma^{2}}=\frac{1}{n}\left(s_{y y}-\mathbf{s}_{x y}^{\prime} \mathbf{S}_{x x}^{-1} \mathbf{s}_{x y}\right) .
$$

Let

$$
\mathbf{A}_{i}=\left(\begin{array}{c}
y_{i} \\
\mathbf{x}_{i}
\end{array}\right)\left(\begin{array}{ll}
y_{i} & \mathbf{x}_{i}^{\prime}
\end{array}\right)=\left(\begin{array}{cc}
y_{i}^{2} & y_{i} \mathbf{x}_{i}^{\prime} \\
\mathbf{x}_{i} y_{i} & \mathbf{x}_{i} \mathbf{x}_{i}^{\prime}
\end{array}\right)
$$

be the $(p+1) \times(p+1)-$ dimensional augmented matrix of the $i^{\text {th }}$ observation by combining the response and the explanatory variables together. Let

$$
\mathbf{A}=\sum_{i=1}^{n} \mathbf{A}_{i}=\left(\begin{array}{ll}
s_{y y} & s_{x y}^{\prime} \\
\mathbf{s}_{x y} & \mathbf{S}_{x x}
\end{array}\right)
$$

be the $(p+1) \times(p+1)-$ dimensional augmented matrix of the entire data set. Then, $s_{y y}, \mathbf{s}_{x y}$, and $\mathbf{S}_{x x}$ can be obtained if $\mathbf{A}$ is derived. Therefore, we have the following theorem.

For cross-validation specifically, Cross-Conformal Prediction (CCP) partitions the training set into $K$ subsets $S_{1}, S_{2}, \ldots, S_{K}$ and calculates the nonconformity scores of the examples in each subset $S_{k}$ and of $\left(x_{l+1}, \tilde{y}\right)$ for each possible label $\tilde{y}$ as

$$
\begin{aligned}
\alpha_{i} & =A\left(\bigcup_{m \neq k} S_{m}, z_{i}\right), z_{i} \in S_{k}, \quad m=1, \ldots, K, \\
\alpha_{l+1}^{\tilde{y}, k} & =A\left(\bigcup_{m \neq k} S_{m}, z_{l+1}^{\tilde{y}}\right), \quad m=1, \ldots, K,
\end{aligned}
$$

where $z_{l+1}^{\tilde{y}}=\left(x_{l+1}, \tilde{y}\right)$. For $z_{l+1}^{\tilde{y}} K$ nonconformity scores $\alpha_{l+1}^{\tilde{y}, k}, k=1, \ldots, K$ are calculated, one with each of the $K$ folds. Now the $p-$ value for each possible label $\tilde{y}$ is computed as

$$
p(\tilde{y})=\frac{\sum_{k=1}^{K}\left|\left\{z_{i} \in S_{k}: \alpha_{i} \geq \alpha_{l+1}^{\tilde{y}, k}\right\}\right|+1}{l+1} .
$$

\section{RESULT ANALYSIS}

All of the computations were carried out by a seventh generation Intel Core $-i 52.8 \mathrm{GHz}$ processor with $8 \mathrm{~GB}$ DDR3 memory. The big data is scanned and mapped, then a scale-model were created based on co-variant matrix, and Gaussian data as a model of big data were taken as a input for analysis. The Gaussian is a continuous distribution

$$
P(y)=\frac{1}{\sigma \sqrt{2 \pi}} e^{-\frac{(y-\mu)^{2}}{2 \sigma^{2}}}
$$

$\mu=$ mean of distribution (also at the same place as mode and median)

$\sigma^{2}=$ variance of distribution

$y$ is a continuous variable $(-\infty \leq y \leq \infty)$

Figure 9 and 8 represent simulation of Gaussian data taken as model of big data.

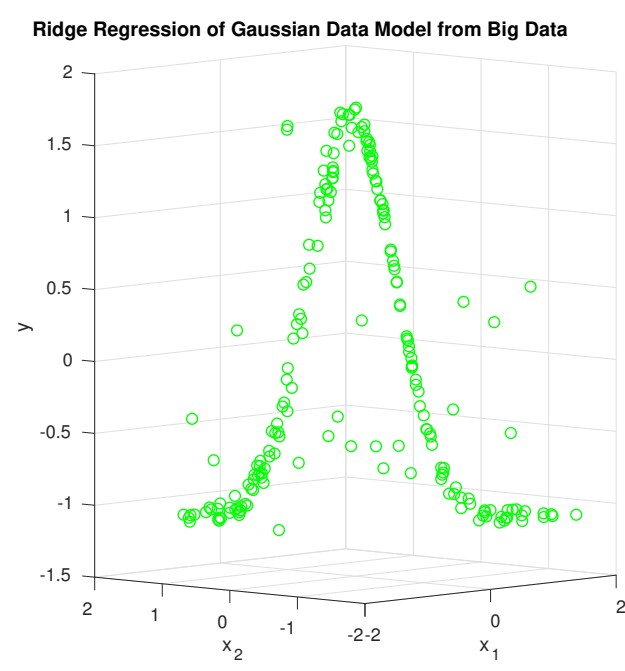

Fig. 8. Proposed Method

The simulation involves one response to be ridge regressive. Data points for the covariates, denoted axis such as $\left(x_{1}, x_{2}, y\right)$, are drawn from a multivariate normal distribution. The points of $x_{1}$ is between 


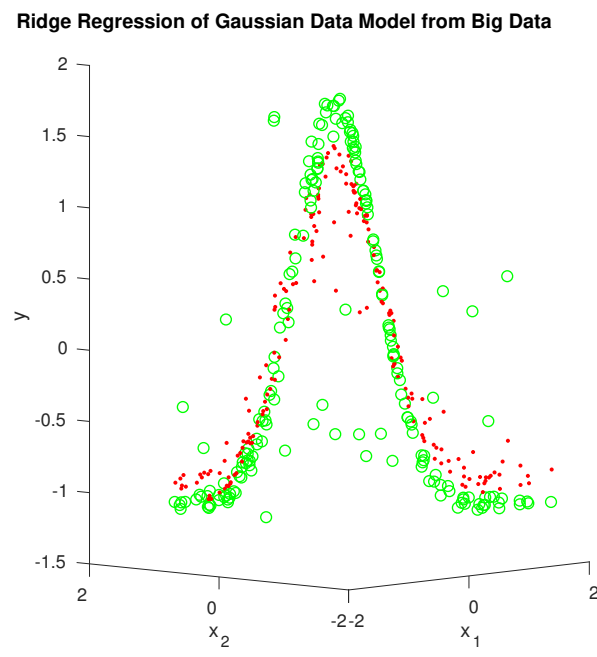

Fig. 9. Proposed Method

( 2 to -2$)$, and points of $x_{2}$ is also (2 to -2$)$ and points of $y$ axis is between $(-1.5$ to 2$)$.

\section{CONCLUSION AND FUTURE WORK}

In this paper, an approach was proposed to overcome the computing load in big data analysis since mostly Ridge Regression as a statistical methods were focused on small sample Gaussian data. In Big data analysis, whole data should be analyzed which are recognized as population in statistics, and this data collection is so large. In the experimental results, the regression parameters estimated by the big data were not different to the parameters by sub data sets. This research contributes to avoid the computing problem in many fields for big data analysis.

This approach can be applied to more diverse methods in statistics such as factor analysis and clustering. More diverse methods of big data sampling are needed in the future works. More advanced combining methods for merging the results of sun data sets can be proposed.

\section{References}

[1] A. J. Bush, "Ridge: A program to perform ridge regression analysis," Behavior Research Methods \& Instrumentation, vol. 12, no. 1, pp. 73-74, Jan 1980.

[2] R. Tibshirani, "Regression shrinkage and selection via the lasso," Journal of the Royal Statistical Society. Series B (Methodological), vol. 58, no. 1, pp. 267-288, 1996.

[3] A. Gepp, M. K. Linnenluecke, T. J. ONeill, and T. Smith, "Big data techniques in auditing research and practice: Current trends and future opportunities," Journal of Accounting Literature, vol. 40, pp. 102 - 115, 2018.

[4] Q. Gao and T. C. Lee, "High-dimensional variable selection in regression and classification with missing data," Signal Processing, vol. 131, pp. 1-7, 2017.

[5] B. Efron, T. Hastie, I. Johnstone, and R. Tibshirani, "Least angle regression,” Ann. Statist., no. 2, pp. 407-499, 04.
[6] J. S. Vitter, "Algorithms and data structures for external memory." Found. Trends Theor. Comput. Sci., vol. 2, no. 4, pp. 305-474, 2006.

[7] J. Dean and S. Ghemawat, "Mapreduce: Simplified data processing on large clusters," pp. 137-150, 012004.

[8] H. Karloff, S. Suri, and S. Vassilvitskii, "A model of computation for mapreduce," in Proceedings of the Twenty-first Annual ACM-SIAM Symposium on Discrete Algorithms, ser. SODA '10, 2010, pp. 938-948.

[9] M. R. Thakare, S. W. Mohod, , and A. N. Thakare, "Various data-mining techniques for big data," IJCA Proceedings on International Conference on Quality Up-gradation in Engineering, Science and Technology, vol. ICQUEST 2015, no. 8, pp. 9-13, October 2015, full text available.

[10] M. Enea, "Fitting linear models and generalized linear models with large data sets in r," Statistical Methods for the Analysis of Large Datasets: book of short papers, pp. 411-414, 2009.

[11] J. Polo, D. Carrera, Y. Becerra, M. Steinder, and I. Whalley, "Performance-driven task co-scheduling for mapreduce environments," in 2010 IEEE Network Operations and Management Symposium - NOMS 2010, April 2010, pp. 373-380.

[12] A. Fernndez, S. del Ro, V. Lpez, A. Bawakid, M. J. del Jesus, J. M. Bentez, and F. Herrera, "Big data with cloud computing: an insight on the computing environment, mapreduce, and programming frameworks," Wiley Interdisciplinary Reviews: Data Mining and Knowledge Discovery, vol. 4, no. 5.

[13] D. Miner and A. Shook, MapReduce Design Patterns: Building Effective Algorithms and Analytics for Hadoop and Other Systems, 1st ed. O'Reilly Media, Inc., 2012.

[14] P. Ma and X. Sun, "Leveraging for big data regression," Wiley Interdisciplinary Reviews: Computational Statistics, vol. 7, no. 1.

[15] S. Guha, R. Hafen, J. Rounds, J. Xia, J. Li, B. Xi, and W. S. Cleveland, "Large complex data: divide and recombine (d and r) with rhipe," Stat, vol. 1, no. 1.

[16] N. Lin and R. Xi, "Aggregated estimating equation estimation." Stat. Interface, vol. 4, no. 1, pp. 73-83, 2011.

[17] B. Gupta, A. Rawat, A. Jain, A. Arora, and N. Dhami, "Analysis of various decision tree algorithms for classification in data mining," International Journal of Computer Applications, vol. 163, no. 8, pp. 15-19, Apr 2017.

[18] R. V. Hogg and A. T. Craig, Introduction to mathematical statistics.(5", edition). Upper Saddle River, New Jersey: Prentice Hall, 1995.

[19] S. Jun and S.-J. L.-B. Ryu, "A divided regression analysis for big data," International Journal of Software Engineering and Its Applications, vol. 9, no. 5, 2015.

[20] L. R. Nair and S. D. Shetty, "Research in big data and analytics: An overview," International Journal of Computer Applications, vol. 108, no. 14, pp. 19-23, December 2014, full text available.

[21] B.-W. Chen, S. Rho, L. T. Yang, and Y. Gu, "Privacypreserved big data analysis based on asymmetric imputation kernels and multiside similarities," Future Generation Computer Systems, vol. 78, no. Part 2, pp. 859 - 866, 2018. 
[22] P. Goldar, Y. Rai, and S. Kushwaha, "A review on parallelization of big data analysis and processing," International Journal of Emerging Technology in Computer Science \& Electronics (IJETCSE), vol. 23, no. 4, pp. 60-65, August 2016.

[23] H. Geng, HADOOP TECHNOLOGY. Wiley Telecom, 2017, pp. 816-

[24] D. Vohra, Using Apache Hadoop. Berkeley, CA: Apress, 2016, pp. 117-130.

[25] W. Q. Meeker and Y. Hong, "Reliability meets big data: Opportunities and challenges," Quality Engineering, vol. 26, no. 1, pp. 102-116, 2014.

[26] Y. Mao and W. Min, "Storage and accessing small files based on hdfs," in Proceedings of International Conference on Computer Science and Information Technology, S. Patnaik and X. Li, Eds. New Delhi: Springer India, 2014, pp. 565-573.

[27] S. G. Edward and N. Sabharwal, Introducing MongoDB. Berkeley, CA: Apress, 2015, pp. 25-28.

[28] L. Vokorokos, M. Uchnr, and A. Bal, "Mongodb scheme analysis," in 2017 IEEE 21st International Conference on Intelligent Engineering Systems (INES), Oct 2017, pp. $000067-$ 000070.

[29] D. Vohra, Using Apache Spark. Berkeley, CA: Apress, 2016, pp. 219-228.

[30] T. Zhang and B. Yang, "An exact approach to ridge regression for big data," Computational Statistics, vol. 32, no. 3, pp. 909-928, Sep 2017.

[31] J. Haworth, J. Shawe-Taylor, T. Cheng, and J. Wang, "Local online kernel ridge regression for forecasting of urban travel times," Transportation Research Part C: Emerging Technologies, vol. 46, no. Supplement C, pp. 151 - 178, 2014.

[32] H. Xue, Y. Zhu, and S. Chen, "Local ridge regression for face recognition," Neurocomputing, vol. 72, no. 4, pp. 1342 - 1346, 2009, brain Inspired Cognitive Systems (BICS 2006) / Interplay Between Natural and Artificial Computation (IWINAC 2007).

[33] A. E. Hoerl and R. W. Kennard, "Ridge regression: Biased estimation for nonorthogonal problems," Technometrics, vol. 12, no. 1, pp. 55-67, 1970.

[34] H. Zhan and S. Xu, "Adaptive ridge regression for rare variant detection," PLOS ONE, vol. 7, 082012.

[35] R. L. Obenchain, "Classical f-tests and confidence regions for ridge regression," Technometrics, vol. 19, no. 4, pp. 429-439, 1977.

[36] J. Fan, F. Han, and H. Liu, "Challenges of big data analysis," National Science Review, vol. 1, no. 2, pp. 293-314, 2014.

[37] X. Shen, M. Alam, F. Fikse, and L. Rönnegård, "A novel generalized ridge regression method for quantitative genetics," Genetics.

[38] Q.-T. Zhang, Y. Liu, W. Zhou, and Z.-W. Yang, "A sequential regression model for big data with attributive explanatory variables," Journal of the Operations Research Society of China, vol. 3, no. 4, p. 475.

[39] J. H. Friedman, "Data mining and statistics: What's the connection?" Computing Science and Statistics, vol. 29, no. 1, pp. 3-9, 1998.
[40] Y. Benjamini and M. Leshno, "Statistical methods for data mining," in Data Mining and Knowledge Discovery Handbook. Springer, 2005, pp. 565-587.

[41] F. Z. Maksood and G. Achuthan, "Analysis of data mining techniques and its applications," International Journal of Computer Applications, vol. 140, no. 3, pp. 6-14, April 2016, published by Foundation of Computer Science (FCS), NY, USA.

[42] A. Mohammadighavam, N. Rajabpour, and A. Naserasadi, "A survey on data mining approaches," International Journal of Computer Applications, vol. 36, no. 6, pp. 14-18, December 2011, full text available.

[43] J. W. Emerson and M. J. Kane, "Don't drown in the data," Significance, vol. 9, no. 4, pp. 38-39, 2012. 